\title{
Triplanar Model for the Gap and Penetration Depth in YBCO
}

\author{
C. O'Donovant and J. P. Carbotte \\ Department of Physics \& Astronomy, McMaster University, \\ Hamilton, Ontario, Canada L8S $4 M 1$
}

(October 10, 1996)

\begin{abstract}
$\mathrm{YBaCuO}_{7}$ is a trilayer material with a unit cell consisting of a $\mathrm{CuO}_{2}$ bilayer with a $\mathrm{CuO}$ plane of chains in between. Starting with a model of isolated planes coupled through a transverse matrix element, we consider the possibility of intra as well as interplane pairing within a nearly antiferromagnetic Fermi liquid model. Solutions of a set of three coupled BCS equations for the gap exhibit orthorhombic symmetry with $s$ - as well as $d$-wave contributions. The temperature dependence and $a-b$ in plane anisotropy of the resulting penetration depth is discussed and compared with experiment.

preprint: cond-mat/9612106
\end{abstract}

\section{INTRODUCTION}

Within the unit cell of $\mathrm{YBa}_{2} \mathrm{Cu}_{3} \mathrm{O}_{7}$, there are two $\mathrm{CuO}_{2}$ planes and a single set of $\mathrm{CuO}$ chains oriented along the $b$-axis. The chains break the tetragonal symmetry of the system and make it orthorhombic. The role of the chains is not yet fully understood but it is clear the they lead to a large in-plane anisotropy in various properties such as the value of the zero temperature penetration depth and the DC conductivity etc. 1- 7] For example, in optimally doped $\mathrm{YBa}_{2} \mathrm{Cu}_{3} \mathrm{O}_{6.95}$, the penetration depth along the $a$-axis at $T=0$ is $\lambda_{a}(0) \sim 1600 \AA$ while that along the $b$-axis is $\lambda_{b}(0) \sim 1030 \AA$. [2] At $300 \mathrm{~K}$ the ratio of the DC resistivities $\rho_{b} / \rho_{a}$ is larger than a factor of two. These large anisotropies would not exist in a purely tetragonal system. The role of the chains in YBCO has been investigated before using a two plane model [8] as well as a plane-chain model. [9, 10] While these calculations have given us considerable insight, such a model is not fully realistic. Here we want to explicitly introduce the trilayer nature of the system. We start with a pair of isolated $\mathrm{CuO}_{2}$ planes and a set of chains ( $\mathrm{CuO}$ plane). They are assumed to be coupled through a transverse matrix element, $t_{1}$, between the chain and each of the planes and $t_{2}$ between the two planes. A set of three coupled BCS equations are used to describe the pairing with intra- as well as interband coupling. For the pairing potential, we use the form of the magnetic susceptibility introduced in the phenomenological nearly antiferromagnetic Fermi liquid model of Monien, Millis and Pines 11 (MMP) which we multiply by a dimensionless parameter $g_{\alpha \beta}$ which allows us to vary at will the strength of the diagonal (in plane) and off diagonal (interplane) couplings. Here the indices $\alpha \beta$ range over the three subbands. Numerical solutions of these equations using a fast Fourier transform technique give solutions which are an admixture of $d, s_{\circ}$ and $s$ symmetry. For the tetragonal crystal point group, these three symmetries would belong to two different irreducible representations and would, in general, not mix. But because of the existence of the chains, the system possesses reduced symmetry although, in most cases considered, nodes remain on the Fermi surface and the linear low temperature variation of the penetration depth remains. In section II, we give formalism. Solutions of the gap equations are discussed in section III along with results for the penetration depth. A brief conclusion is included in section IV.

\section{FORMALISM}

Consider a three plane per unit cell system with energies $\varepsilon_{\mathbf{k}, \alpha}$. Here $\alpha=1,2,3$ is an index that enumerates the three planes; two $\mathrm{CuO}_{2}$ which are identical to each other and one $\mathrm{CuO}$ plane consisting of chains oriented along the $\mathrm{b}$-direction. The momentum index $\mathbf{k}$ is restricted to the two dimensional $\mathrm{CuO}_{2}$ Brillouin zone which has tetragonal symmetry. A tight binding model will be used to describe these isolated planes of the form: [12 14

$$
\begin{aligned}
\varepsilon_{\mathbf{k}, \alpha}= & -2 t_{\alpha}\left[\left(1+\epsilon_{\alpha}\right) \cos \left(k_{x}\right)+\left(1-\epsilon_{\alpha}\right) \cos \left(k_{y}\right)\right. \\
& \left.-2 B_{\alpha} \cos \left(k_{x}\right) \cos \left(k_{y}\right)-\left(2-2 B_{\alpha}-\mu_{\alpha}\right)\right],
\end{aligned}
$$

where $t_{\alpha}$ is the first neighbour hopping parameter, $\epsilon_{\alpha}$ an orthorhombic distortion which can be used to describe the chains, $B_{\alpha}$ is the second neighbour hopping in units of $t_{\alpha}$, the $k_{i}$ are in units of reciprocal lattice vectors and $\mu_{\alpha}$ is related to the chemical potential and determines the filling. Here it will be treated as a parameter. In what follows, the $t_{\alpha}$ 's will be taken to be the same for simplicity and denoted by $t_{0}$. The three dimensional nature of the system is introduced through transverse matrix elements, $t_{1}$, between the $\mathrm{CuO}_{2}$ planes and the $\mathrm{CuO}$ chains and $t_{2}$ directly between the $\mathrm{CuO}_{2}$ bilayer. This changes the energies $\varepsilon_{\mathbf{k}, \alpha}$ to band energies $\tilde{\varepsilon}_{\mathbf{k}, \alpha}$ which we then use in the BCS gap equations assuming singlet pairing between equal and opposite momentum pairs. Including intraband (on diagonal) as well as interband (off diagonal) pairing through the 2-body potential $V_{\mathbf{k}, \mathbf{k}^{\prime}, \alpha \beta}$ which describes scattering from $\mathbf{k}$ to $\mathbf{k}^{\prime}$ with $\alpha$ and $\beta$ band indices the gap in the $\alpha^{\text {th }}$ band $\Delta_{\mathbf{k}, \alpha}$ satisfies the BCS equations: 10] 


$$
\Delta_{\mathbf{k}, \alpha}=\frac{1}{\Omega} \sum_{\mathbf{k}^{\prime}, \beta} V_{\mathbf{k}, \mathbf{k}^{\prime}, \alpha \beta} \frac{\Delta_{\mathbf{k}^{\prime}, \alpha}}{2 E_{\mathbf{k}^{\prime}, \alpha}} \tanh \left(\frac{E_{\mathbf{k}^{\prime}, \alpha}}{2 k_{\mathrm{B}} T}\right),
$$

In equation (2), $\Omega$ is the crystal volume, $k_{\mathrm{B}}$ Boltzmann's constant, $T$ temperature and

$$
E_{\mathbf{k}, \alpha}= \pm \sqrt{\tilde{\varepsilon}_{\mathbf{k}, \alpha}^{2}+\Delta_{\mathbf{k}, \alpha}^{2}}
$$

are the quasiparticle energies of the $\alpha^{\text {th }}$ band in the superconducting state. The single particle normal state band energies for a two layer model come from the Hamiltonian:

$$
H=\left[\begin{array}{ll}
\varepsilon_{1} & t_{1} \\
t_{1} & \varepsilon_{2}
\end{array}\right]
$$

with eigenvalues:

$$
\tilde{\varepsilon}_{1,2}=\left(\frac{\varepsilon_{1}+\varepsilon_{2}}{2}\right) \pm \sqrt{\left(\frac{\varepsilon_{1}-\varepsilon_{2}}{2}\right)^{2}+t_{1}^{2}}
$$

and for the three layer model come from the Hamiltonian:

$$
H=\left[\begin{array}{ccc}
\varepsilon_{1} & t_{1} & t_{2} \\
t_{1} & \varepsilon_{2} & t_{1} \\
t_{2} & t_{1} & \varepsilon_{1}
\end{array}\right]
$$

with eigenvalues:

$$
\begin{aligned}
\tilde{\varepsilon}_{1}= & \varepsilon_{1}-t_{2} \\
\tilde{\varepsilon}_{2}= & \frac{\varepsilon_{1}+\varepsilon_{2}+t_{2}}{2} \\
& +\frac{1}{2} \sqrt{\left(\varepsilon_{1}-\varepsilon_{2}\right)^{2}+2 t_{2}\left(\varepsilon_{1}+\varepsilon_{2}+t_{2}\right)+8 t_{1}^{2}}
\end{aligned}
$$

and

$$
\begin{aligned}
\tilde{\varepsilon}_{3}= & \frac{\varepsilon_{1}+\varepsilon_{2}+t_{2}}{2} \\
& -\frac{1}{2} \sqrt{\left(\varepsilon_{1}-\varepsilon_{2}\right)^{2}+2 t_{2}\left(\varepsilon_{1}+\varepsilon_{2}+t_{2}\right)+8 t_{1}^{2}}
\end{aligned}
$$

which are respectively the solid, dashed and dotted curves in Fig. 5 .

The set of three coupled equations (2) for the gap's $\Delta_{\mathbf{k}, \alpha}$ in the $\alpha^{\text {th }}$ band $(\alpha=1,2,3)$ are explicit once the pairing potentials $V_{\mathbf{k}, \mathbf{k}^{\prime}, \alpha \beta}$ are specified. Different models could be used. Here for simplicity and to be specific, we will assume that the superconducting condensation proceeds through the spin susceptibility $\chi_{\mathbf{k}, \mathbf{k}^{\prime}}$ which for the nearly antiferromagnetic Fermi liquid is peaked at the commensurate wave vector $(\pi, \pi)$ at the corner of the Brillouin zone. So as not to introduce new parameters, here we will use the form of $\chi_{\mathbf{k}, \mathbf{k}^{\prime}}$ introduced through a fit to NMR data by Millis, Monien and Pines (MMP). 11] Their susceptibility and consequent pairing potential has the form:

$$
V_{\mathbf{k}, \mathbf{k}^{\prime}, \alpha \beta}=g_{\alpha \beta} \frac{-t_{\circ}}{1+\xi_{\circ}^{2}\left|\mathbf{k}-\mathbf{k}^{\prime}-\mathbf{Q}\right|^{2}},
$$

where the scale on $V_{\mathbf{k}, \mathbf{k}^{\prime}, \alpha \beta}$ has been set by $t_{\circ}$ for convenience and $g_{\alpha \beta}$ are dimensionless parameters that determine the overall size of the pairing as well as the relative amount of diagonal $(\alpha=\beta)$ and off diagonal $(\alpha \neq \beta)$ coupling desired. In all our calculations, the relative sizes of the $g_{\alpha \beta}$ will be chosen at will with absolute value adjusted to get a critical temperature of $100 \mathrm{~K}$ typical for the oxide superconductors. The other parameters in (7) are also specified and fixed at values given by MMP. $\xi_{\circ}=2.5 \AA$ is the magnetic coherence length and $\mathbf{Q}$ the commensurate wave vector $(\pi, \pi)$ in the 2 dimensional $\mathrm{CuO}_{2}$ Brillouin zone.

In writing down the set of equations (2), we have assumed that the coherence length transverse to the planes is short so that the gap can have different values in the different bands $\tilde{\varepsilon}_{\mathbf{k}, \alpha}$. The model still allows for a single gap, applicable to all three planes, to exist. The appropriate limit is $g_{\alpha \beta}$ independent of $\alpha$ and $\beta$ in which case $\Delta_{\mathbf{k}, \alpha}$ on the left hand side of (2) is clearly independent of $\alpha$ and a single gap parameter describes the entire system.

Numerical solution of the set of equations (2) using fast Fourier transforms makes no assumption on the symmetry of the resulting gap parameters $\Delta_{\mathbf{k}, \alpha}$ as a function of momentum $\mathbf{k}$ in the 2 dimensional Brillouin zone. In our work, we find that these solutions consist of a mixture of $d_{x^{2}-y^{2}}, s_{\circ}$ and $s_{x^{2}+y^{2}}$ symmetries with higher harmonics as well as the more familiar lowest ones which are

$$
\begin{aligned}
s_{\circ} \sim \eta_{\mathbf{k}}^{\left(s_{\circ}\right)} & \equiv 1 \\
s_{x^{2}+y^{2}} \sim \eta_{\mathbf{k}}^{\left(s_{x}\right)} & \equiv \cos \left(k_{x}\right)+\cos \left(k_{y}\right) \\
d_{x^{2}-y^{2}} \sim \eta_{\mathbf{k}}^{(d)} & \equiv \cos \left(k_{x}\right)-\cos \left(k_{y}\right) .
\end{aligned}
$$

We are now ready to present results including the temperature dependence of the penetration depth $\lambda(T)$ for various model parameters. To obtain the penetration depths, we have adopted the following approximate procedure. The lowest harmonics represented by (8a) to (8d) are projected out of our numerical solutions and these are used in all further calculations. This neglects higher harmonic contributions but is an adequate procedure since we are mainly interested in qualitative results and trends. In any case, our model for the pairing interaction (7) is simplified and is not believed to be accurate. We use a BCS approach while it is necessary to go to an Eliashberg formulation to get quantitative results. This goes beyond the scope of this work.

For completeness, the approximate formula for the penetration depth employed here is:

$$
\lambda_{i j}^{-2}=\frac{4 \pi e^{2}}{c^{2}} \frac{1}{\Omega} \sum_{\mathbf{k}, \alpha} v_{\alpha, i} v_{\alpha, j}\left(\frac{\partial f\left(\tilde{\varepsilon}_{\mathbf{k}, \alpha}\right)}{\partial \tilde{\varepsilon}_{\mathbf{k}, \alpha}}-\frac{\partial f\left(E_{\mathbf{k}, \alpha}\right)}{\partial E_{\mathbf{k}, \alpha}}\right)
$$

where the constant $c$ is the speed of light, $v_{\alpha, i}$ is the $i^{\text {th }}$ component of the electron velocity in the $\alpha^{\text {th }}$ band

$$
v_{\alpha, i} \equiv \frac{1}{\hbar} \frac{\partial \tilde{\varepsilon}_{\mathbf{k}, \alpha}}{\partial k_{i}}
$$


and $f(x)$ is the Fermi-Dirac distribution given by

$$
f(x) \equiv\left(e^{\tilde{\varepsilon}_{\mathbf{k}, \alpha} / k_{\mathrm{B}} T}+1\right)^{-1}
$$

We are now in a position to present results.

\section{RESULTS}

Before presenting results for the trilayer model, it is instructive to consider a bilayer consisting of a $\mathrm{CuO}_{2}$ plane with tetragonal symmetry and a set of $\mathrm{CuO}$ chains directed along the $b$ axis which has lower orthorhombic symmetry. This difference in symmetry has a drastic effect on the solution of the BCS gap equation. To illustrate this, we take a model band structure:

$$
\left\{t_{\circ}, \epsilon_{\alpha}, B_{\alpha}, \mu_{\alpha}\right\}=\left\{\begin{array}{rrrr}
50.0 & 0.0 & 0.45 & 0.51 \\
50.0 & 1.0 & 0.00 & 1.2
\end{array}\right\}
$$

with $t_{\circ}$ in $\mathrm{meV}$ and the other parameters are dimensionless. We also consider the pairing potential to be of the MMP type and of equal magnitude in both chains and plane. So as to have a single critical temperature, we also include a smaller off diagonal pairing $g_{\alpha \beta} \neq 0$ for $\alpha \neq \beta$. Specifically, we take:

$$
g_{\alpha \beta}=\left[\begin{array}{rr}
38.75 & 1.0 \\
1.0 & 38.75
\end{array}\right]
$$

Solutions of the BCS equations using fast Fourier transforms give an admixture of $s_{\circ}, s_{x^{2}+y^{2}}$ and $d_{x^{2}-y^{2}}$ which all belong to the same irreducible representation for an orthorhombic system. The gap amplitudes are

$$
\Delta_{\mathbf{k}, \alpha}=\left[\begin{array}{lll}
-0.196 & -0.467 & 9.482 \\
-0.644 & -0.182 & 1.482
\end{array}\right]\left[\begin{array}{c}
\eta_{\mathbf{k}}^{\left(s_{\circ}\right)} \\
\eta_{\mathbf{k} x}^{\left(s_{x}\right)} \\
\eta_{\mathbf{k}}^{(d)}
\end{array}\right]
$$

with the $\eta_{\mathbf{k}}^{(\cdot)}$ as given in (8a-8c). We note that in the $\mathrm{CuO}_{2}$ plane, the $d_{x^{2}-y^{2}}$ component is dominant with a small admixture of $s_{\circ}$ and $s_{x^{2}+y^{2}}$ having the opposite sign (out of phase by $\pi$ ). Although the same MMP interaction as used in the $\mathrm{CuO}_{2}$ planes has been assumed to also apply on the $\mathrm{CuO}$ chains, the very different symmetry of the quasi one-dimensional Fermi surface has led to a gap on the chains which is smaller by an order of magnitude. The $d_{x^{2}-y^{2}}$ component has the same phase as it has in the planes but it is now not much larger than its $s_{\circ}$ and $s_{x^{2}+y^{2}}$ parts. In Fig. 11(b) (middle upper frame) and (e) (middle lower frame), we show the $\mathrm{CuO}_{2}$ and $\mathrm{CuO}$ Fermi surfaces (dashed lines), respectively, as well as the contour of gap zeros (solid lines). In Fig. 1(b), the Fermi surface has tetragonal symmetry but the gap zeros have nevertheless moved off the main diagonals (solid curve) because of the admixture into the mainly $d_{x^{2}-y^{2}}$ gap function of $s_{\circ}$ and $s_{x^{2}+y^{2}}$ components. As these are negative in equation (14), the zeros cross the Fermi surface at an angle $(\theta)$ measured clockwise from the negative $k_{y}$ axis which is slightly larger than $45^{\circ}$. This is seen more clearly in Fig. 1 (a) where we show the absolute value of the gap on the Fermi surface as a function of $\theta$ for the plane. Note that the gap does not display full tetragonal symmetry in that the zeros are displaced from $45^{\circ}$ and $135^{\circ}$ and that the gap at $90^{\circ}$ is smaller than it is at $0^{\circ}$ and $180^{\circ}$.

For the chain case, the gap zeros (solid curves in Fig. 1(e)) are displaced much more off the main diagonals and as illustrated in Fig. 11(d) the value of the absolute value of the gap as a function of $\theta$ along the quasi onedimensional chain Fermi surface is very small. Note that this is a result of the orthorhombicity (ie, geometry of the Fermi surface) of the chain Fermi surface as the pairing potential has been assumed to have the same size and form in the chains as in the tetragonal planes. For the given value of pairing interaction, the gap ratio $2 \Delta_{\max } / T_{\mathrm{c}}$ is 3.86 in the plane while it has been reduced to 0.47 in the chains. Finally, it is important to note that in Fig. 11(d), the gap on the Fermi surface starts at a finite value of $\theta$ reflecting the geometry of the nearly linear chain Fermi surface. The very small energy scale exhibited by the gap in the chains leads directly to a very steep slope in the resulting $b$-axis penetration depth as seen in the solid curve of Fig. 1(f). The contribution of the chains to the $a$-axis penetration depth is by contrast (dashed line) very small. This is quite different from the contribution of the plane to the penetration depth illustrated in Fig. 1 1 (c). In this case, the nearly tetragonal symmetry of the Fermi surface leads to very similar contributions for the temperature dependence of the $a$ - (dashed) and $b$ (solid) direction penetration depth. The small differences between these two curves as to absolute value of $\lambda^{-2}(0)$ at zero temperature is due to the orthorhombic symmetry of the dispersion (equation (11)). Unfortunately, these small differences are not measured directly in an experiment which probes the sum of contributions from chains and planes, ie, Fig. 1(f) and 11(c). This sum is shown in Fig. 2 where it is compared with experiments [1] (circles). The agreement is poor although the order of the zero temperature slopes is correct, i.e. it is steeper in the chain direction (b-direction). The large discrepancy can be traced directly to the small energy scale found in the chains in this model and we can conclude that the data favours a gap which is of the same magnitude in the chains as it is in the planes. Finally, we note that $\lambda_{a} / \lambda_{b} \simeq 1.4$ in our calculation, which is reasonably close to (but not exactly) the measured value.

It is easy to modify our model so that chains and planes have the same gap value. A single gap results for $\Delta_{\mathbf{k}, \alpha}$ independent of $\alpha$ if all diagonal and off diagonal elements of the pairing potential are taken to have the same value, i.e. $g_{\alpha \beta}=$ constant for any $\alpha$ and $\beta$. In Fig. 3, we illustrate results obtained with the same band parameters as used in our previous model but now with $g_{\alpha \beta}=24.7$ for all $\alpha$ and $\beta$ in which case a single gap results which 
has the form:

$$
\Delta_{\mathbf{k}}=\left[\begin{array}{lll}
-1.83 & -0.288 & 10.09
\end{array}\right]\left[\begin{array}{c}
\eta_{\mathbf{k}}^{\left(s_{\circ}\right)} \\
\eta_{\mathbf{k}}^{\left(s_{x}\right)} \\
\eta_{\mathbf{k}}^{(d)}
\end{array}\right] .
$$

This gap has mainly $d$-wave character with a small admixture of $s_{\circ}$ and $s_{x^{2}+y^{2}}$ so that the gap zeros (solid curve) in Fig. B(b) and 1 (e) have moved off the main diagonal as in the previous example. Now these zero contours are the same in both chains and planes. The absolute value of the gap (on the Fermi surface) as a function of $\theta$ are shown in Fig. 3(a) and (d) for plane and chain, respectively, and the maximum value of $2 \Delta / k_{\mathrm{B}} T_{\mathrm{c}}$ is 4.5 in the planes and 3.76 in the chains, much more comparable in value than was the case in the first model considered. It is to be noted that over the entire Brillouin zone the gaps in the planes and chains are not different but, of course, the Fermi surface geometry is totally different in these two cases. In particular, we note that on the Fermi surface the gap in the chains starts at a finite angle $\theta$ near $45^{\circ}$ and that there is no Fermi surface at small values of $\theta$.

The smaller value of the maximum gap found in the chains as opposed to the planes will lead to a steeper slope of the low temperature dependence of the inverse square of the penetration depth in the chain direction as seen in Fig. 4 where we compare with experiments. While theory predicts somewhat steeper slopes, qualitative agreement with experiment is obtained in this simple model.

We turn next to results obtained for the trilayer model with $\mathrm{CuO}_{2}$ planes on both sides of a $\mathrm{CuO}$ chain plane. The transverse coupling between $\mathrm{CuO}_{2}$ and $\mathrm{CuO}$ plane is denoted by $t_{1}$ and $t_{2}$ is a direct transverse coupling between the two $\mathrm{CuO}_{2}$ planes of the same unit cell. For $\left\{t_{\circ}, t_{1}, t_{2}\right\}=\{50,30,15\}$ in meV the resulting Fermi surfaces obtained from equation 6 are shown in Fig. 同 where:

$$
\left\{\begin{array}{cccc}
t_{\circ} & \epsilon_{\alpha} & B_{\alpha} & \mu_{\alpha}
\end{array}\right\}=\left\{\begin{array}{cccc}
50.0 & 0.0 & 0.45 & 0.51 \\
50.0 & 1.0 & 0.0 & 1.2 \\
50.0 & 0.0 & 0.45 & 0.51
\end{array}\right\},
$$

with $t_{\circ}$ in meV. The chain Fermi surface, denoted by the dashed line, is quasi one dimensional and has only orthorhombic symmetry. The two $\mathrm{CuO}_{2}$ plane Fermi surfaces are shown as dotted (odd) and solid (even) curves. Only the even band Fermi surface has tetragonal symmetry while the odd band is flatter along $k_{x}$ than it is along $k_{y}$. This orthorhombicity will lead to a different $a$ - and $b$-direction contributions to the penetration depth from the $\mathrm{CuO}_{2}$ planes themselves and this will now be described.

A first set of results that is instructive is illustrated in Fig. 6. The chains are assumed not to contribute directly to the penetration depth but only provide an agency whereby the $\mathrm{CuO}_{2}$ plane acquires orthorhombic symmetry. This is accomplished easily if no pairing is assumed to act on the chains be it diagonal or off diagonal. That is, we take for the pairing potential the matrix:

$$
g_{\alpha \beta}=\left[\begin{array}{ccc}
36.8 & 0.0 & 15.0 \\
0.0 & 0.0 & 0.0 \\
15.0 & 0.0 & 36.8
\end{array}\right]
$$

What is critical in equation (17) is that the second row is all zero so the chains remain normal by arrangement. In this case, we obtain a gap of the form:

$$
\Delta_{\mathbf{k}, \alpha}=\left[\begin{array}{ccc}
1.81 & -1.46 & 9.96 \\
0.0 & 0.0 & 0.0 \\
0.59 & -0.80 & 10.88
\end{array}\right]\left[\begin{array}{c}
\eta_{\mathbf{k}}^{\left(s_{\circ}\right)} \\
\eta_{\mathbf{k}}^{\left(s_{x}\right)} \\
\eta_{\mathbf{k}}^{(d)}
\end{array}\right] .
$$

The gap on even (top row) and odd (bottom row) band is mainly $d$-wave like with $s$-components an order of magnitude smaller. The contours of gap zeros are illustrated as the solid curves in Fig. 6(b) and (e) for odd and even bands, respectively. The Fermi contour is the dashed curve in each case. The absolute value of the gap $(|\Delta|)$ as a function of angle $\theta$ along the Fermi surface are given in Fig. F(a) and (d) for odd and even band, respectively. In both cases, the gap zeros occur slightly before $\theta=45^{\circ}$ and beyond $135^{\circ}$ so that the gap does not have tetragonal symmetry even in the even band with a tetragonal Fermi surface. Also, the maximum gap on the Fermi surface is not quite the same in the two bands and is $2 \Delta / k_{\mathrm{B}} T_{\mathrm{c}}=4.5$ and 3.69 for even and odd cases, respectively. While these values are not very different, the contributions of even and odd bands to the $a$ - and $b$-direction penetration depths are very different. The odd band with orthorhombic Fermi surface gives a factor of 2 difference in the value of the zero temperature penetration depth as shown in Fig. 6(c). The solid curve applies along the $b$ direction and the dashed curve along the $a$-direction. For the even Fermi surface, which has tetragonal symmetry, the situation is very different as illustrated in Fig. f(f) where it is seen that solid (along $b$-direction) and dashed curve (along $a$-direction) are nearly the same (the slight difference is due to the difference in the $a$ and $b$ lattice parameters). Finally, in Fig. 7, we compare results for the $a$ - (dashed) and $b$-direction (solid) penetration depth with experimental results. Again the curves are correctly ordered as to value of slope at zero temperature and the agreement is qualitative. It is as good as that shown in Fig. (6, although the physics of the two cases is quite different. In Fig. 7, the chains are normal by assumption and do not contribute directly to the condensate density in the $a$ - or $b$-directions. The in plane anisotropy is due mainly to the odd band Fermi surface which has been made orthorhombic through its coupling to the chains and much less importantly to the $s$ admixture in the gap function. Thus, we are dealing mainly with a band structure effect which dominates and overwhelms any gap symmetry effects.

As a second illustration of a triplanar model, we show in Fig. 8 results for model parameters chosen so that the chains also become superconducting but by virtue of off diagonal coupling and not because there is intrinsic 
pairing in the chains themselves. That is $g_{22}$ is assumed to be zero. Specifically, we assume:

$$
g_{\alpha \beta}=\left[\begin{array}{ccc}
44.4 & 10.0 & 0.0 \\
10.0 & 0.0 & 10.0 \\
0.0 & 10.0 & 44.4
\end{array}\right]
$$

while the band parameters remain the same as in the previous cases. In Fig. 8(b), (e) and (h), we show, respectively, odd, chain and even band Fermi surface as the dashed curves and contours of gap zeros is the solid curves. The gap solution is:

$$
\Delta_{\mathbf{k}, \alpha}=\left[\begin{array}{ccc}
1.21 & -1.35 & 7.77 \\
0.65 & -0.34 & 3.79 \\
-0.59 & 0.15 & 11.17
\end{array}\right]\left[\begin{array}{c}
\eta_{\mathbf{k}}^{\left(s_{\circ}\right)} \\
\eta_{\mathbf{k}}^{\left(s_{x}\right)} \\
\eta_{\mathbf{k}}^{(d)}
\end{array}\right] .
$$

The absolute values of the gaps as a function of angle $\theta$ along odd, chain and even Fermi surface are shown in fig. 8(a), (d) and (g). Because no pairing was assumed to act in the chains, the gap is smallest in frame (d) with maximum $2 \Delta / k_{\mathrm{B}} T_{\mathrm{c}}$ equal to 1.88 as opposed to 4.58 and 2.98 for even and odd band, respectively. Frames (c), (f) and (l) show that odd orthorhombic band makes a very different contribution to the $b$-direction (solid curve) penetration depth than to the $a$-direction; the difference in zero temperature value being of order 2 . The differences are even more pronounced in the case of the chains which contribute very little to the $a$-direction (dashed curve) while the even tetragonal Fermi surface makes almost equal contribution to $a$ - and $b$-directions. Because of the small energy scale present on the chains, which can be traced to the assumption $g_{22}=0$, i.e. zero pairing on the chains, this model will not lead to good agreement with experiment. To achieve this, a final model is considered with the same single gap for both $\mathrm{CuO}_{2}$ planes and for the chains. If we take all $g_{\alpha \beta}=$ constant $=18.4$, we obtain a single gap

$$
\Delta_{\mathbf{k}}=\left[\begin{array}{lll}
-0.363 & -0.502 & 10.14
\end{array}\right]\left[\begin{array}{c}
\eta_{\mathbf{k}}^{\left(s_{\circ}\right)} \\
\eta_{\mathbf{k}}^{\left(s_{x}\right)} \\
\eta_{\mathbf{k}}^{(d)}
\end{array}\right] .
$$

Detailed results are illustrated in the nine frames of Fig. 9. In Fig. 9 (b), (e) and (h), the gap zeros are all the same (solid curves) yet because the Fermi contours (dashed curves) are different, the gap as a function of angle along the Fermi surfaces are different as shown in Fig. 9 (a), (d) and (g) for odd, chain and even Fermi surfaces, respectively. The maxima in $2 \Delta / k_{\mathrm{B}} T_{\mathrm{c}}$ are respectively $4.39,4.55$ and 4.07. Resulting contributions to the $a$ (dashed) and $b$ - (solid) direction penetration depth are found in Fig. 9 (c), (f) and (i). The odd orthorhombic Fermi surface again contributes about twice as much to $b$ - and to $a$ - direction at low temperature while the even one contributes equally. The chains mainly contribute to the $b$-direction. The sum of all contributions are shown in Fig. 10 and compared with experiment. The agreement is satisfactory with $a$-direction curve (dashed) above the $b$ direction (solid) as observed. The ratio $\lambda_{a} / \lambda_{b}$ was $\sim 1.6$ in the calculations, close to the observed value.

\section{CONCLUSIONS}

We have presented results of calculations for the temperature dependence of the in plane penetration depth in a trilayer model of $\mathrm{YBa}_{2} \mathrm{Cu}_{3} \mathrm{O}_{7}$ which includes two $\mathrm{CuO}_{2}$ planes and a set of $\mathrm{CuO}$ chains oriented along the $b$-direction. Transverse hopping matrix elements couple the three planes together leading to three coupled bands in which the gap parameters is an admixture of $d_{x^{2}-y^{2}}$, $s_{x^{2}+y^{2}}$ and $s_{\circ}$ symmetries. While the $s$ and $d$ symmetry types belong to two different irreducible representations of the tetragonal crystal point group, they belong to the same representation in the orthorhombic case which is the case we are dealing with here because of the presence of the chains. Nevertheless, it is found that the penetration depth remains linear in temperature at low temperature as a result of nodes in the gap crossing the Fermi contours. To compare with experiment, the ratio of $\lambda_{a} / \lambda_{b}$ was kept near the observed value in all our calculations. With this constraint, it was found that the observed normalized temperature variation of the penetration depth in $a$ - and $b$-directions is difficult to understand in models where no intraplane pairing is introduced in the chains even if a large amount of interplane pairing is considered. The most natural explanation of the data is obtained when the pairing interaction is assumed not to depend significantly on sub-band and is taken to be independent of $\alpha$ and $\beta$, i.e. $V_{\mathbf{k}, \mathbf{k}^{\prime}, \alpha \beta} \equiv V_{\mathbf{k}, \mathbf{k}^{\prime}}$. Also the zero temperature anisotropy in $\lambda_{a, b}$ can be traced to the orthrhombicity of the band structure which resides in the chains and hybridized planes.

\section{ACKNOWLEDGEMENT}

Work supported in part by the Canadian Institute for Advanced Research (CIAR) and the Natural Sciences and Engineering Research Council of Canada (NSERC). We have benefited from conversations with W. Atkinson.

$\dagger \quad$ Electronic address: odonovan@mcmaster.ca

[1] D.N. Basov, R. Liang, D.A. Bonn, W.N. Hardy, B. Dabrowski, M. Quijada, D.B. Tanner, J.P. Rice, D.M. Ginsberg and T. Timusk Phys. Rev. Lett. 74, 598 (1995).

[2] K. Zhang, D.A. Bonn, S. Kamal, R. Liang, D.J. Baar, W.N. Hardy, D. Basov and T. Timusk, Phys. Rev. Lett. 73, 2484 (1994). 


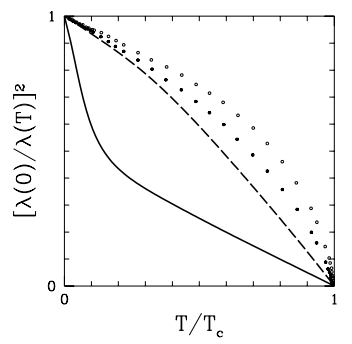

FIG. 2. Total contribution to the $a$ - (dashed) and $b$-directions (solid) as a function of temperature to the inverse square of the penetration depth normalized to its zero temperature value. The dots are experimental values [1] with $b$-direction falling below the $a$-direction. The $b$-direction theoretical curve is much too steep at low temperature because of the small energy scale for the chain gap.

[3] D.A. Bonn, S. Kamal, K. Zhang, R. Liang and W.N. Hardy, J. Phys. Chem. Solids 56, 1941 (1995).

[4] T.A. Friedmann, M.W. Fabin, J. Giapintzakis, J.P. Rice and D.M. Ginsberg, Phys. Rev. B42, 6217 (1990).

[5] Y. Iye in Physical Properties of High Temperature Superconductors Vol III, p. 285 (edited by D.M. Ginsberg, World Scientific, Singapore (1992).

[6] R. Gagnon, C. Jupien and L. Taillefer, Phys. Rev. B50, 3458 (1994).

[7] K. Takenaka, K. Mizuhashi, H. Takage and S. Uchida, Phys. Rev. B50, 6534 (1994).

[8] W.A. Atkinson and J.P. Carbotte, Phys. Rev. B 51, 1161 (1995); Phys. Rev. B 51, 16371 (1995).

[9] W.A. Atkinson and J.P. Carbotte, Phys. Rev. B 52, 6894 (1995); Phys. Rev. B 52, 10601 (1995).

[10] C. O'Donovan and J.P. Carbotte, to appear in Phys. Rev. B 54, (1996).

[11] A.J. Millis, H. Monien and D. Pines, Phys. Rev. B 42, 167 (1990).

[12] C. O'Donovan and J.P. Carbotte, Phys. Rev. B 52, 4548 (1995).

[13] C. O'Donovan, D. Branch, J.P. Carbotte and J. Preston, Phys. Rev. B 51, 6588 (1995).

[14] C. O'Donovan and J.P. Carbotte, Physica C 252, 87 (1995).

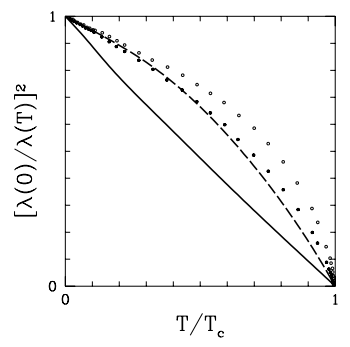

FIG. 4. Total contribution to the $a$ - (dashed) and $b$-directions (solid) as a function of temperature to the inverse square of the penetration depth normalized to its zero temperature value. The dots are experimental values [1] with the $b$-direction falling below the $a$-direction. The agreement is good and value of $\lambda_{a} / \lambda_{b} \approx 1.4$ is also in reasonable but not exact agreement with experiment.

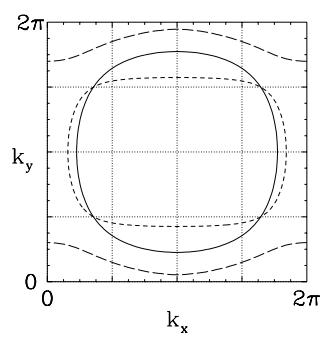

FIG. 5. The three Fermi surfaces in the triplanar model of YBCO. The chain like band (dashed curve) is nearly one dimensional, the odd band Fermi surface has orthorhombic symmetry (dotted curve) due to its interaction with the chains and the even band (solid curve) remains tetragonal.

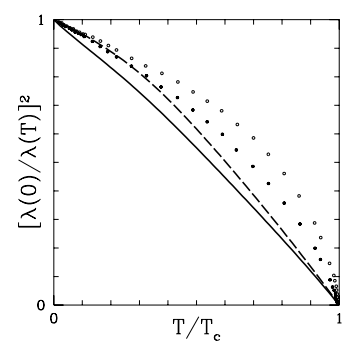

FIG. 7. Total contributions to the inverse square of the penetration depth normalized to its zero temperature value as a function of temperature. The solid curve is for the $b$-direction and the dashed for the $a$-direction. The dots are the experimental data [1] with $b$-direction curve below the $a$-direction curve. Results are for the trilayer model of Figs. 5 and 6 in which it is assumed that there is no pairing on the chains on or off diagonal so they remain normal and do not contribute directly to the condensate. 
(a)

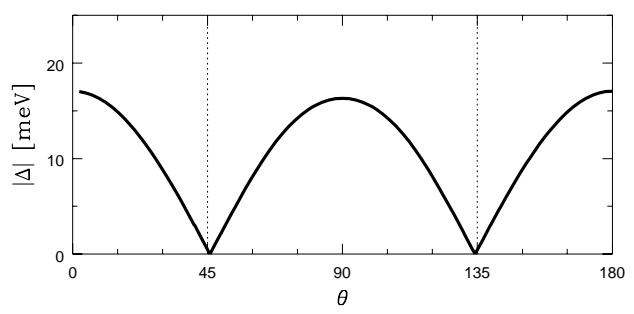

(d)

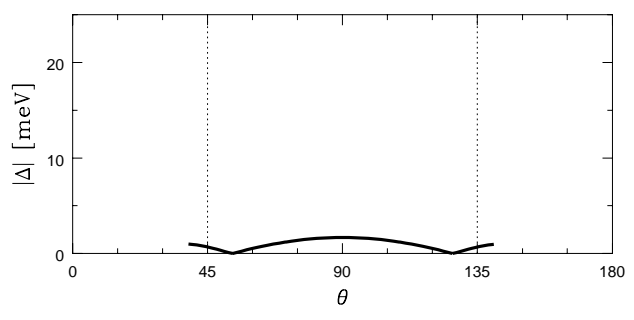

(b)

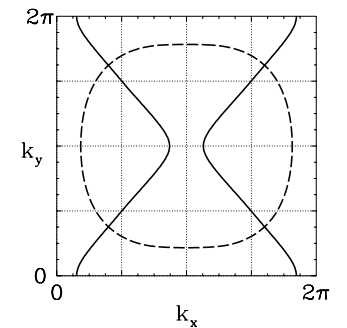

(e)

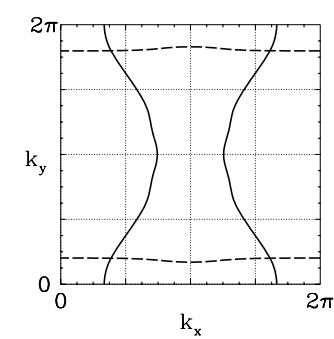

(c)

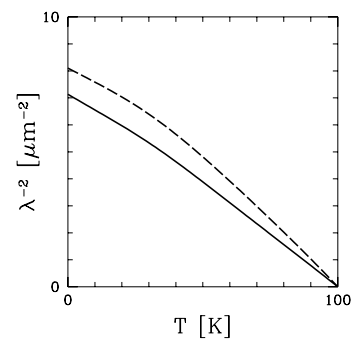

(f)

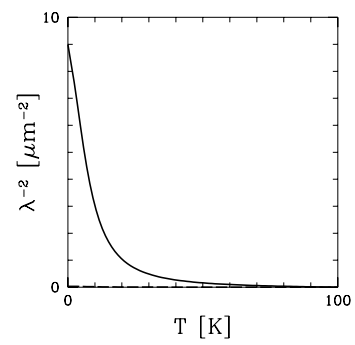

FIG. 1. (a) and (d) are the absolute value of gap as a function of angle $\theta$ measured clockwise from the $k_{x}$ axis along the Fermi surface for the $\mathrm{CuO}_{2}$ plane and $\mathrm{CuO}$ chains, respectively. (b) and (e) are Fermi surface contours (dashed line) and gap zeros (solid line) while (c) and (f) are the contributions to the inverse square of the penetration depth $\lambda^{-2}(T)$ as a function of temperature along $a$ - (dashed curve) and $b$-direction (solid curve). Note that the chains Fig. (d), (e) and (f) contribute almost exclusively to the $b$-direction. The same pairing was included in chains and plane (equation 13 yet note the very small energy scale for the gap in the chains (d) as compare to the gap in the plane (a). This is due to the quasi-one dimensional nature of the chain Fermi surface. Note also that the low energy scale in the chains leads to the sharp drop in penetration depth in $b$-direction with increasing temperature seen in (f). 
(a)

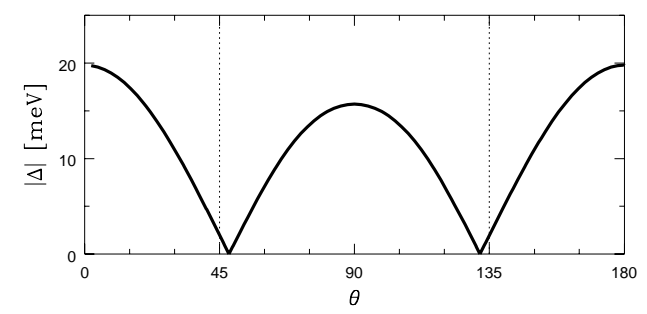

(d)

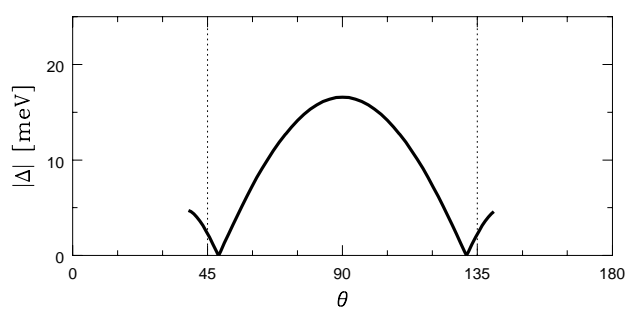

(b)

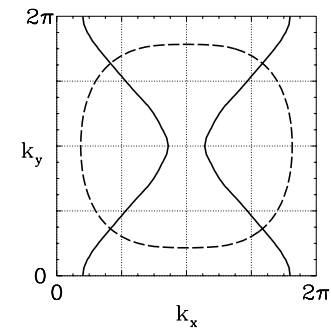

(e)

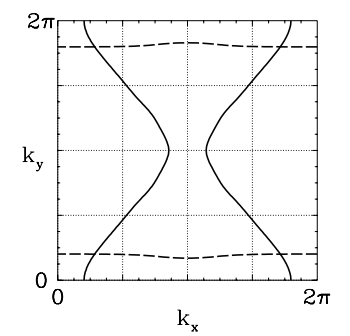

(c)

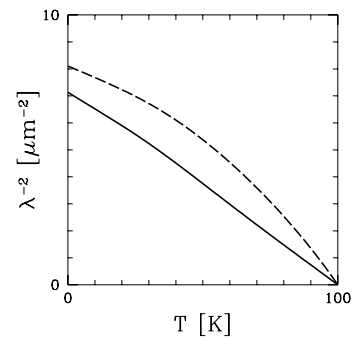

(f)

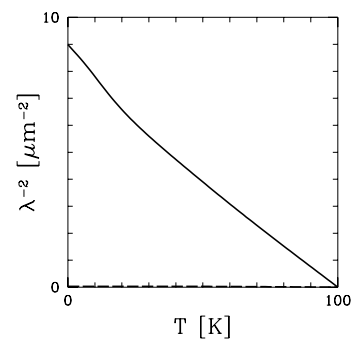

FIG. 3. Frame (a), (b) and (c) apply to the $\mathrm{CuO}_{2}$ planes while (d), (e) and (f) apply to the CuO chains with a nearly one dimensional Fermi surface. The pairing interaction is assumed to be the same on chains and planes and off diagonal so that a single gap results. Figs. (b) and (e) give the Fermi surface contours (dashed curve) and the gap zeros (solid curves) which are the same in both frames but different from pure $d$-wave which would be lines on the main diagonals in the Brillouin zone. Frame (a) and (d) give the absolute value of the gap as a function of angle $\theta$ along the Fermi surface measured clockwise from the $k_{x}$ axis. Frame (c) and (f) give partial contributions to the inverse square of the penetration depth as a function of temperature along $a$-direction (dashed) and $b$-direction (solid). Note that the zeros in the gap are not at $45^{\circ}$ and $135^{\circ}$ as they would be in pure $d$-wave (Fig. (a) and (d)). 
(a)

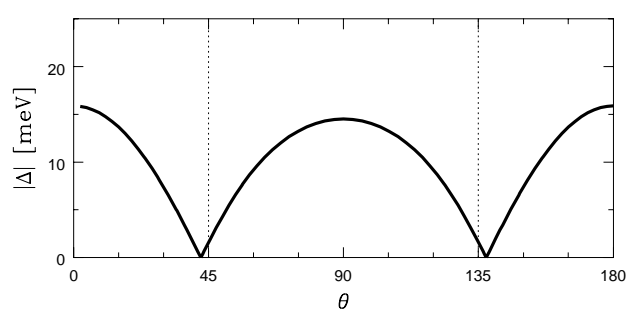

(d)

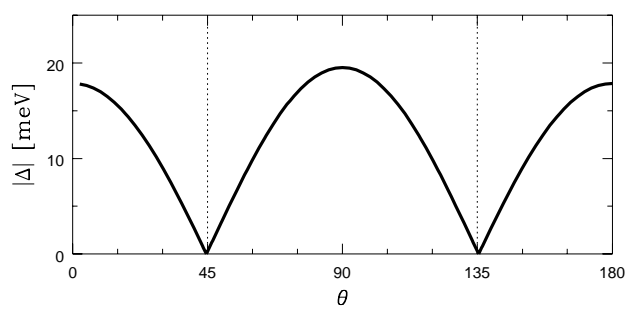

(b)

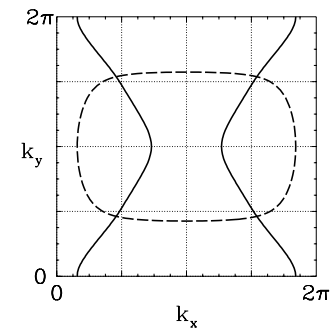

(e)

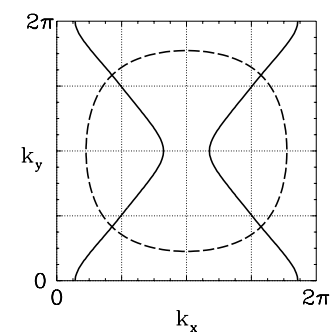

(c)

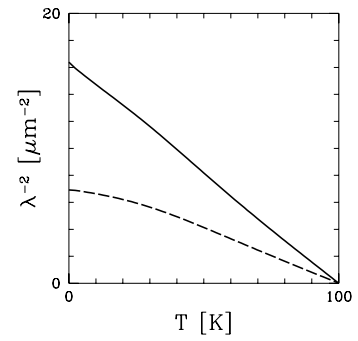

(f)

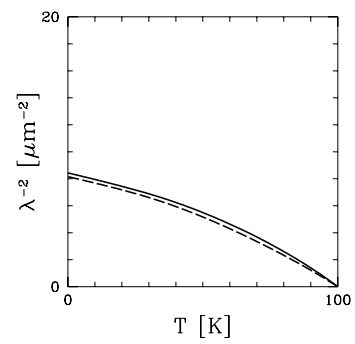

FIG. 6. Results for the triplanar model illustrated in Fig. 月 assuming no pairing diagonal or off diagonal on the chains which then remain normal. Frame (a), (b) and (c) apply to the odd band (orthorhombic symmetry) while (d), (e) and (f) apply to the even band (tetragonal symmetry). The first two frames (a) and (d) illustrate the absolute value of the gap along the Fermi surface as a function of angle $\theta$ measured clockwise from the $k_{x}$ axis. These curves do not have full tetragonal symmetry and the zeros are displaced somewhat from the ideal $d_{x^{2}-y^{2}}$ case which would be at $45^{\circ}$ and $135^{\circ}$. Frame (b) and (e) give the Fermi surface (dashed line) and gap nodes (solid line) for odd and even plane (tetragonal), respectively. The final frames (c) and (f) give partial contributions to the inverse square of the penetration depth as a function of temperature for $a$ - (dashed) and $b$ - (solid) direction. Note that the odd (orthorhombic) band gives a contribution to the $b$-direction which is about twice its contribution to the $a$-direction, while the even band (tetragonal) gives nearly the same contribution in both directions. In the model, the ratio $\lambda_{a} / \lambda_{b} \approx 1.3$. 
(a)

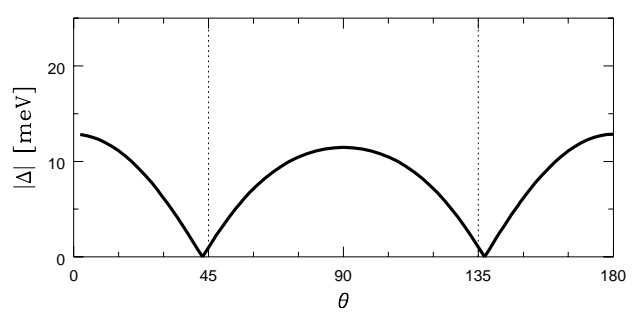

(d)

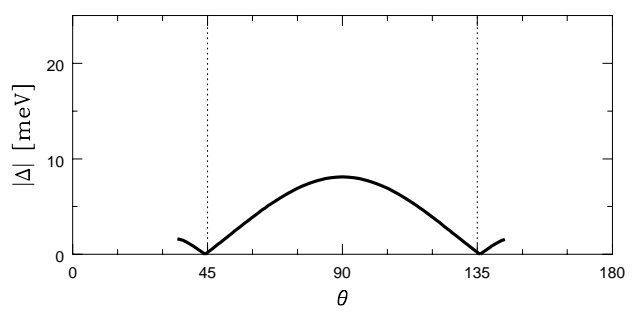

$(\mathrm{g})$

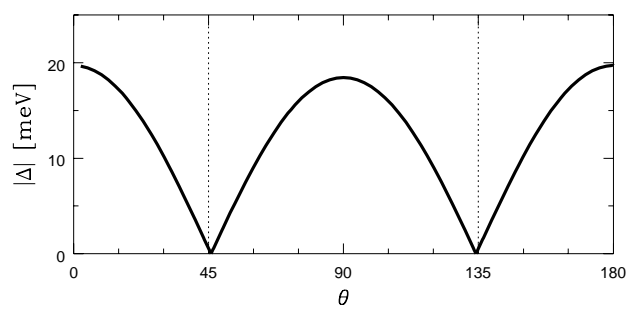

(b)

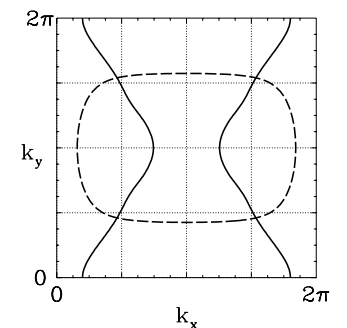

(e)

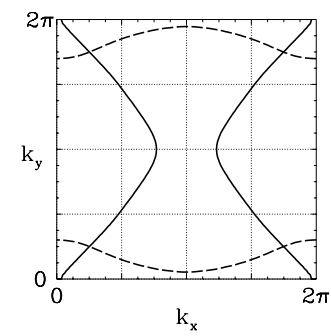

(h)

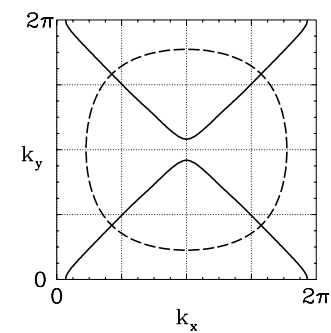

(c)

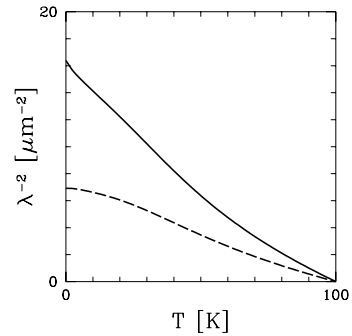

(f)

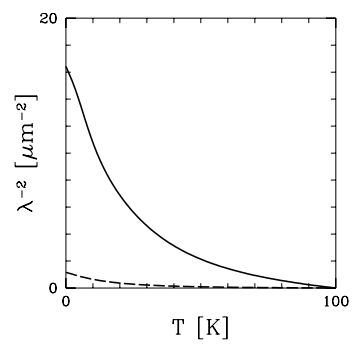

(i)

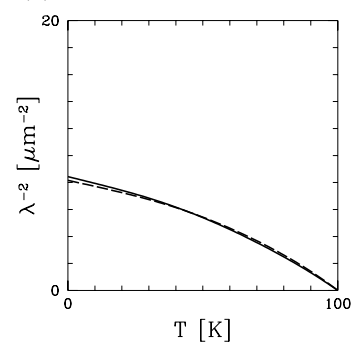

FIG. 8. Results for a triplanar model with some off diagonal pairing on the chains but with $g_{22}=0$, i.e. no direct pairing in the chains themselves. The chains become superconducting but with a small gap amplitude. Top frames apply to the odd (orthorhombic) $\mathrm{CuO}_{2}$ band, the middle three to the chain $(\mathrm{CuO})$ band and the lower three frames to the even (tetragonal) $\mathrm{CuO}_{2}$ band. Frames (a), (d) and (g) give the absolute value of the gap as a function of angle $\theta$ along the Fermi surface measured clockwise from the $k_{x}$ axis. Note the lack of tetragonal symmetry in the gap and the shift off $45^{\circ}$ and $135^{\circ}$ of the gap nodes. Frame (b), (e) and (h) give the Fermi surface (dashed curve) and gap zero contours (solid curve). The odd $\mathrm{CuO}_{2}$ plane band is orthorhombic as are the chains while the even $\mathrm{CuO}_{2}$ band Fermi surface retains tetragonal symmetry. Contributions to the inverse square of the penetration depth as a function of temperature are given in frames (c), (f) and (i) for $a$-direction (dashed) and $b$-direction (solid). The odd orthorhombic band contributes almost twice as much to the $b$ - as to the $a$-direction while the even tetragonal band makes almost equal contributions. The chains contribute mainly in the $b$ - direction. 
(a)

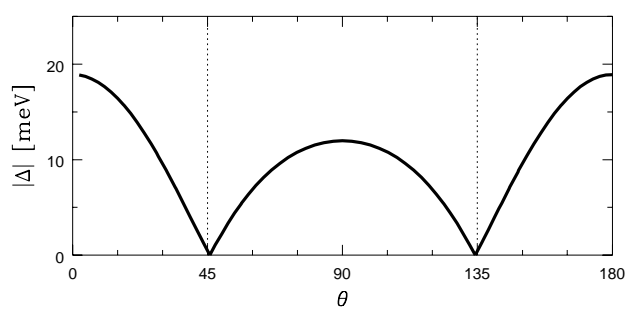

(d)

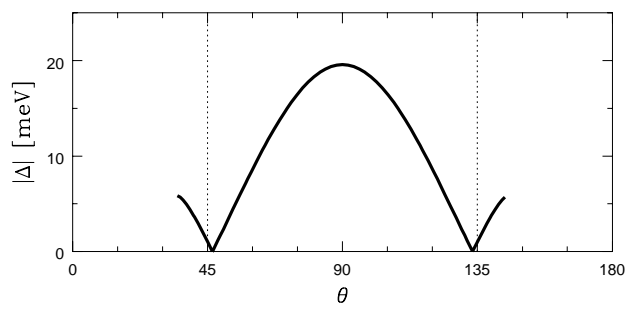

$(\mathrm{g})$

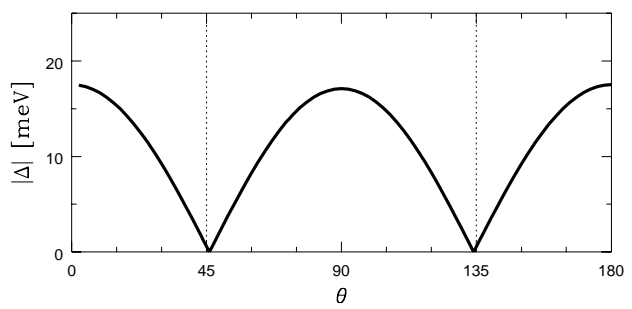

(b)

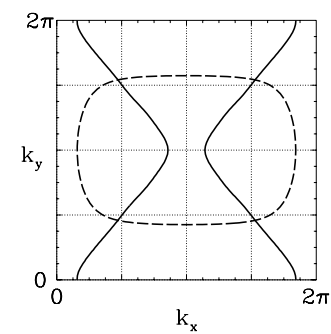

(e)

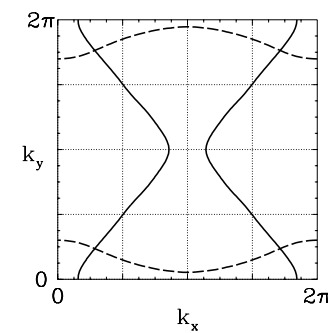

(h)

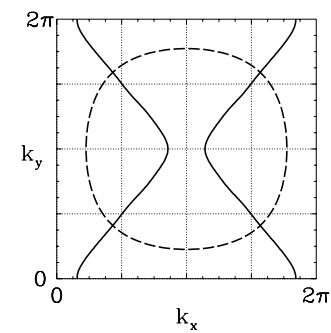

(c)

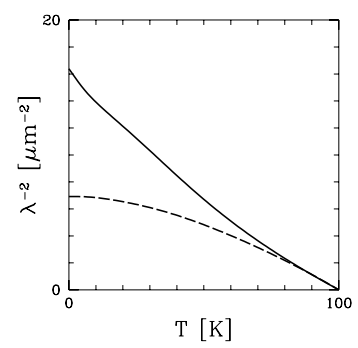

(f)

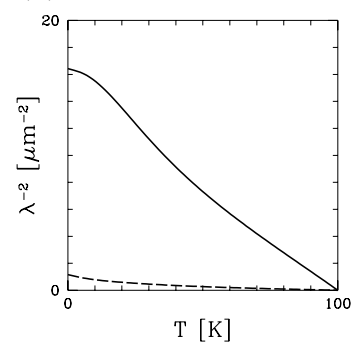

(i)

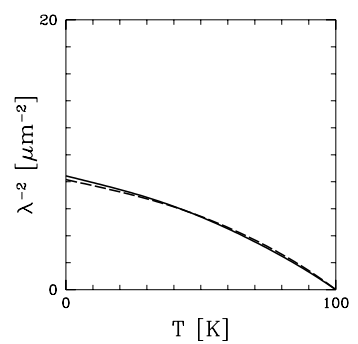

FIG. 9. Results for a trilayer model with pairing interaction having the same value on and off diagonal, i.e. $g_{\alpha \beta}=$ constant for all $\alpha$ and $\beta$. This assumption ensures that a single gap applies to both the $\mathrm{CuO}_{2}$ planes and the $\mathrm{CuO}$ chain. The three upper frames apply to the odd $\mathrm{CuO}_{2}$ orthorhombic band, the middle three to the $\mathrm{CuO}$ chain band and the lower three to the even $\mathrm{CuO}_{2}$ (tetragonal) band. Frames (a), (d) and (g) gives the gap on the Fermi surface as a function of angle $\theta$ measured clockwise from the $k_{x}$-axis. Note the lack of tetragonal symmetry and the "missing parts" in the chain case which reflects the geometry of the quasi one dimensional Fermi surface. Frame (b), (e) and (h) give the Fermi contours (dashed line) for odd $\mathrm{CuO}_{2}$ (orthorhombic) band, chain like $\mathrm{CuO}$ band and even $\mathrm{CuO}_{2}$ (tetragonal) band. The solid curve are the gap zero contours and are the same in all three figures. Frames (c), (f) and (i) give the contributions to the inverse square of the penetration depth as a function of temperature for the $a$-direction (dashed) and the $b$-direction (solid). Note that the odd orthorhombic band contributes almost twice as much to the $b$ - as it does to the $a$-direction in contrast to the even (tetragonal) band which makes nearly equal contributions. The chains contribute mainly to the $b$-direction. In this model part of the $a$ - $b$ asymmetry in the penetration depth comes from the orthorhombic nature of the even plane Fermi surface of frame (b). 


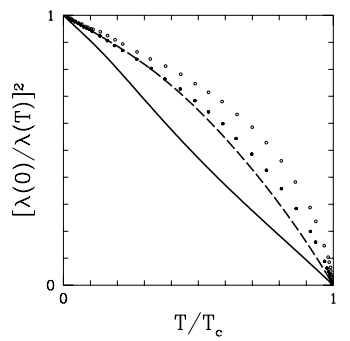

FIG. 10. Total contributions to the inverse square of the penetration depth normalized to its zero temperature value as a function of temperature. The solid curve applies to the $b$-direction and dashed to the $a$-direction. The dots are experimental data [4] with the $b$-direction falling below $a$-direction. The model is that of Fig. 1 with pairing interaction assumed constant (i.e. $g_{\alpha \beta}=$ constant) independent of $\alpha$ and $\beta$ which leads to a single gap for all three planes in the primitive cell. This does not imply that it has $d_{x^{2}-y^{2}}$ symmetry because the bands do not have tetragonal symmetry and the gap contains an admixture of $s_{\circ}$ and $s_{x^{2}+y^{2}}$ although it is still dominantly $d_{x^{2}-y^{2}}$. 